was detected in 35 (47.9\%) patients with 4 (5.5\%) having false-negative results. Overall, the sensitivity of ultrasonography in the detection of hemoperitoneum was $88 \%$; in group R, $87 \%$; group S, $89 \%$; and in group $\mathrm{EM}, 90 \%$. Specificity was in all cases $100 \%$.

Conclusions: Emergent ultrasound examination in detection of hemoperitoneum is equally valid in the hands of various specialists if they are appropriately trained and have the same level of experience.

Key Words: hemoperitoneum; ultrasonograply

\section{Burn Injuries in Traffic Accidents: \\ Are They Avoidable?}

A. Schinnerl; G. Kroesen; M. Baubin; W. Voelckel

Institute for Emergency and Disaster Medicine, Department of Anesthesia and Intensive Care, Innsbruck, Austria

According to Austria's Central Office for Statistics, from 1989 to 1995 , approximately 60 fatal accidents occurred each year due to fire. It is estimated that up to $50 \%$ of these deaths were due to traffic accidents. In 1993, the Institute for Safety in Vienna registered 25 burn victims (411 treatment days) from traffic accidents compared with 19 patients (523 treatment days) in 1994. Treatment of a patient with a burn index of 80 , costs approximately DM 8,700 per day (ATS 62,000). The lasting visible damage from disfigurement following burns, which are not life-threatening, is demonstrated by a prominent former Austrian racing pilot and presentday airline owner.

Very few burns in traffic accidents occur as a result of the primary explosion: most are suffered through cable fires following automobile body damage which then ignite flammable gases and liquids. A simple, automatically controlled foam extinguishing system could primarily extinguish the source of the fire and thus, help prevent deaths. Saving human lives, and secondarily, the economic benefits are convincing arguments in favor of implementing the foam system.

Key Words: burns; costs; deaths; explosion; fire

\section{Re-operation following Abdominal Trauma \\ João Ricardo Ribas, Jr; E Savino Gasparini \\ Rio de Janeiro, Brazil}

Objectives: Review of medical charts from patients who underwent abdominal re-intervention in the emergency surgery department to identify underlying mechanism of trauma, initial treatment, complications, clinical and imagining data, morbidity, and normality.

Patients and Methods: From November 1991 through November 1996, 1,246 abdominal surgical procedures were performed at the emergency surgery department in the Miguel Couto County Hospital, Rio de Janeiro, Brazil. Thirty charts representing patients who required 77 abdominal operations were selected for review.

Results: We divided abdominal re-operations into planned and unplanned interventions. Seven patients had their operations planned for definitive treatment and removal of hemostatic liver packing. Seven patients had developed intra-abdominal abscesses. Five patients suffered evisceration, and four had five intra-abdominal injuries missed at initial exploration, seven patients had an anastomotic failure. One patient had a negative exploration hospitalization period with a high median stay of 36 days and low mortality rate.

Conclusion: Frequent clinical examination was decisive in $60 \%$ of the patients to indicate surgical intervention for abdominal distention and Multiple Organ Failure were the most often used determinants. Those patients operated early had a better outcome, although sometimes it was difficult to determine the best timing.

Key Words: abdominal re-operation; trauma

\section{Thoraco-Abdominal Trauma \\ Savino Gasparini; João Ricardo Ribas, Jr \\ Rio De Janeiro, Brazil}

Objectives: Review of medical charts in order to analyze the results of treatment dispensed to patients with thoraco-abdominal trauma, with emphasis on the occurrence of diaphagmatic hernias.

Patients and Methods: 103 medical charts of patients who sustained thoraco-abdominal trauma from August 1991 until August 1995, including 14 diaphragmatic hernias were reviewed. Age, gender, mechanism of injury, associated injuries, organs injured, treatment, complications, and mortality were analyzed.

Results: Penetrating injuries comprised $61.3 \%$ of the series with gunshot wounds constituting the main injuring agent. One patient was hit by an arrow (uncommon)! The lung was the organ most commonly injured (43.7\%). Diaphragmatic herniation occurred in $13.6 \%$ : 10 were diagnosed in acute period, three in the chronic period, and one during treatment for a complication. One patient in this group was treated through videolaparoscopy. The mortality rate in this sample was $6.8 \%$.

Conclusions: Thoraco-abdominal trauma offers favorable outcome when diagnosed early, even though very often present as serious injuries. Diaphragmatic wounds must be carefully sought, and the correct treatment instituted during the initial exploration.

Key Words: diaphragmatic hernia; thoraco-abdominal trauma

\section{Injuries to the Neck \\ Savino Gasparini; Joäo Ricardo Ribas, Jr. \\ Rio De Janeiro, Brazil}

Objective: To analyze the results of treatment of 76 trauma patients with injuries to the neck who were operated in the Miguel Couto County hospital to compare two different approaches: mandatory and selective exploration.

Patients and Methods: Medical charts of 76 patients with neck injuries who presented at emergency room of Miguel Couto County hospital from July 1990 to July 1995 were reviewed. Age group, gender, mechanism of injury level of injury, diagnostic procedures complications, and mortality were analyzed. 